調査・資料

\title{
大阪府下の市販食肉および食肉加工食品中の PCB および 有機塩素系農薬の残留実態
}

(平成 17 年 12 月 19 日受理)

\author{
松本比佐志* ${ }^{\dagger}$ 桑原克義* 村上保行* 村田 弘*
}

Survey of PCB and Organochlorine Pesticide Residues in Meats and Processed Meat Products Collected in Osaka, Japan

\author{
Hisashi Matsumoto*, ${ }^{\dagger}$, Katsuyoshi Kuwabara*, Yasuyuki Murakami* \\ and Hiroshi Murata*
}

(* Laboratory of Food Chemistry, Division of Food and Drugs, Osaka Prefectural Institute of Public Health: 1-3-69, Nakamichi, Higashinari-ku, Osaka 537-0025, Japan; ${ }^{\dagger}$ Corresponding author)

\begin{abstract}
Residues of PCB and organochlorine pesticides in meats (beef, pork and poultry) and processed meat products collected in Osaka, Japan were surveyed. For 35 years from 1970 to 2004, the concentrations of $\mathrm{PCB}, \mathrm{HCH}$ isomers, DDT analogues and dieldrin in all meats appeared to decline with each passing year. The concentration of each isomer of $\mathrm{HCH}$ in all meats declined rapidly from the early to the late 1970's and showed a gentle decline thereafter; $\beta$-HCH showed the highest residue rate of the isomers in all the time periods surveyed, except for pork and poultry samples in the early 1980's. The concentrations of DDT analogues in all meats also showed sharp declines from the late 1970's to the early 1980's, followed by a smaller decrease thereafter; the main residues were $p, p^{\prime}$-DDD, $p, p^{\prime}$-DDE and $p, p^{\prime}$-DDT, and the residue rate of $p, p^{\prime}$-DDE increased over the years. The residue levels of organochlorine pesticides in processed meat products (raw materials: beef, pork, poultry and mixture of ground beef and pork) were surveyed for 15 years (from 1990 to 2004) and there were no significant differences in the values among the materials. The concentrations of each pesticide in processed meat products tended to be lower than those of the corresponding meat samples, respectively.
\end{abstract}

(Received December 19, 2005)

Key words: 食肉 meat; 食肉加工食品 processed meat product; ポリ塩化ビフェニル PCB; 有機塩素 系農薬 organochlorine pesticide; ヘキサクロロシクロヘキサン HCH; ジクロロジフェニルトリク ロロエタン DDT; ディルドリン dieldrin; ガスクロマトグラフィー-電子捕獲型検出器 GC-ECD

\section{緒言}

PCB (polychlorinated biphenyl) や 1,2,3,4,5,6-hexachlorocyclohexane ( $\alpha, \beta, \gamma$ および $\delta$ 異性体の総和を $\mathrm{HCHs}$ と略す), DDT (1,1,1-trichloro-2,2-bis(4-chlorophenyl)ethane) 関連化合物（総和をDDTs と略す）, dieldrin, heptachlor 抒よびその代謝物の heptachlor epoxide (HCE), hexachlorobenzene (HCB) などの有機塩素系農薬 は，種々の法的規制により日本国内ではその製造・販売・ 使用が禁止されている1), 2). 規制後に長い年月が経過した 近年でも，食品中にはまだ上記の污染物質が高濃度に残留 している事例が魚介類や食肉に見受けられる

\footnotetext{
$\dagger$ 連絡先

* 大阪府立公衆衛生研究所食品医薬品部食品化学課： $\overline{7} 537-$ 0025 大阪市東成区中道 1-3-69
}

の食品の摂取量から計算して污染物質量が許容一日摂取量 (ADI) を超えることはなく, また，上記污染物質の摂取量 は, total diet study 法による摂取量調査であ, ADI より はるかに低い量である99.

しかしながら，近年，これらの合成化学物質の一部のも のに生体のホルモン機構や免疫機能を阻害する恐れのある ことが報告され ${ }^{10)}$ ，その阻害作用は，特に，従来の毒性発 現濃度領域よりさらに低用量領域で生じる可能性が指摘さ れている ${ }^{11}$. これらの污染物質のヒトへの摂取や体内での 蓄積の低減化を図るためには，たとえ低濃度であっても， 食品中の污染物質の残留状況の分析・監視を継続して行う ことが必要と考えられる，そこで，本資料では，大阪府下 で 35 年間（1970 2004 年）にわたって採取された食肉 中の PCB 抒よび有機塩素系農薬と，15 年間（1990～ 
Table 1. Concentration Changes of PCB and Organochlorine Pesticides in Beef Samples Collected on the Market in Osaka, Japan for 35 Years (1970-2004)

\begin{tabular}{|c|c|c|c|c|c|c|c|c|}
\hline \multirow{2}{*}{\multicolumn{2}{|c|}{ Chemicals }} & \multicolumn{7}{|c|}{ Year } \\
\hline & & '70-'74 & '75-'79 & '80-'84 & '85-'89 & '90-'94 & '95-'99 & '00-'04 \\
\hline \multirow{3}{*}{$\mathrm{PCB}$} & $n$ & NT & $7(6)$ & $18(12)$ & $22(7)$ & $22(1)$ & $34(0)$ & $25(0)$ \\
\hline & $\mathrm{Av} \pm \mathrm{SD}(\mathrm{ppb})$ & & $2.6 \pm 1.7^{*}$ & $1.1 \pm 1.1^{\dagger}$ & $0.41 \pm 0.67^{* \#}$ & $0.05 \pm 0.21^{* \dagger}$ & $n d^{* \dagger \#}$ & $\mathrm{nd}^{* \dagger}$ \\
\hline & Range (ppb) & & $n d-5$ & $n d-4$ & nd-2 & $n d-1$ & nd & nd \\
\hline \multirow{3}{*}{$\mathrm{HCH}^{\mathrm{a})}$} & $n$ & $47(46)$ & $26(26)$ & $18(16)$ & $42(20)$ & $42(28)$ & $40(24)$ & $25(6)$ \\
\hline & $\mathrm{Av} \pm \mathrm{SD}(\mathrm{ppb})$ & $2593 \pm 4330^{*}$ & $84 \pm 51^{* \dagger}$ & $24 \pm 22^{* \dagger \#}$ & $5.4 \pm 15^{* \dagger \#}$ & $12 \pm 31^{* \dagger} \AA$ & $4.9 \pm 12^{* \dagger \#}$ & $4.3 \pm 20^{* \dagger \# \S}$ \\
\hline & Range (ppb) & nd-19987 & $12-167$ & nd-85 & nd-92 & nd-161 & nd-51 & nd-99 \\
\hline \multirow{3}{*}{$\mathrm{DDT}^{\mathrm{b})}$} & $n$ & $47(41)$ & $26(26)$ & $18(13)$ & $42(32)$ & $42(21)$ & $40(39)$ & $25(16)$ \\
\hline & $\mathrm{Av} \pm \mathrm{SD}(\mathrm{ppb})$ & $290 \pm 370^{*}$ & $49 \pm 50^{* \dagger}$ & $7.6 \pm 6.9^{* \dagger}$ & $7.3 \pm 10^{* \dagger \#}$ & $6.0 \pm 20^{* \dagger \#}$ & $2.9 \pm 4.1^{* \dagger}$ & $3.3 \pm 7.8^{* \dagger}$ \\
\hline & Range (ppb) & nd-1091 & $1-244$ & nd-22 & nd -52 & nd-119 & nd-26 & nd-39 \\
\hline \multirow{3}{*}{ Dieldrin } & $n$ & $47(14)$ & $26(24)$ & $18(9)$ & $42(8)$ & $42(4)$ & $40(17)$ & $25(6)$ \\
\hline & $\mathrm{Av} \pm \mathrm{SD}(\mathrm{ppb})$ & $13 \pm 37^{*}$ & $11 \pm 12^{* \dagger}$ & $5.9 \pm 13^{\dagger \#}$ & $0.60 \pm 1.4^{\dagger}$ & $0.36 \pm 1.3^{\dagger \# \S}$ & $0.73 \pm 1.1^{\dagger \S}$ & $0.44 \pm 1.2^{\dagger}$ \\
\hline & Range (ppb) & nd -180 & nd -50 & nd-55 & nd-6 & nd -7 & nd-5 & $\mathrm{nd}-6$ \\
\hline \multirow{3}{*}{$\mathrm{HCE}^{\mathrm{c})}$} & $n$ & NT & $26(0)$ & $18(0)$ & $42(4)$ & $42(2)$ & $40(6)$ & $25(1)$ \\
\hline & $\mathrm{Av} \pm \mathrm{SD}(\mathrm{ppb})$ & & nd & nd & $0.33 \pm 1.1$ & $0.14 \pm 0.64$ & $0.35 \pm 1.2$ & $0.04 \pm 0.20$ \\
\hline & Range (ppb) & & nd & nd & nd-5 & $n d-3$ & nd-7 & nd-1 \\
\hline \multirow{3}{*}{$\mathrm{HCB}^{\mathrm{d})}$} & $n$ & NT & $26(4)$ & $18(0)$ & $42(5)$ & $42(0)$ & $40(6)$ & $25(1)$ \\
\hline & $\mathrm{Av} \pm \mathrm{SD}(\mathrm{ppb})$ & & $0.62 \pm 1.6$ & nd & $0.14 \pm 0.42$ & nd & $0.18 \pm 0.44$ & $0.04 \pm 0.20$ \\
\hline & Range (ppb) & & nd-6 & nd & nd-2 & nd & nd-2 & nd-1 \\
\hline
\end{tabular}

The concentration of each chemical (PCB, on a whole basis; each pesticide, on a fat basis) is shown as the average (Av) \pm standard deviation (SD). $n$ : number of samples; those in which chemicals were detected are shown in parentheses. NT: not tested. nd: not detected $(<1 \mathrm{ppb})$; the value was statistically treated as zero. Aldrin and endrin were not detected in any samples. ${ }^{\text {a) }}$ Total amounts of $\alpha-, \beta$-, $\gamma$ - and $\delta$-hexachlorocyclohexanes. ${ }^{\text {b) }}$ Total amounts of $p, p^{\prime}$-DDD, $o, p^{\prime}$-DDE, $p, p^{\prime}$-DDE, $o, p^{\prime}$ DDT and $p, p^{\prime}$-DDT. ${ }^{c)}$ Total amounts of heptachlor and heptachlor epoxide. ${ }^{\text {d) }}$ Hexachlorobenzene.

$*,+, \#, s$ : Of the values marked by the same symbols, the one in the leftmost column is significantly different from the others $(p<0.05)$.

2004 年) に採取された食肉加工食品中の有機塩素系農薬 の残留実態を分析調査し，その年度推移をまとめたので報 告する.

\section{実 験方 法}

\section{1. 試薬および試料}

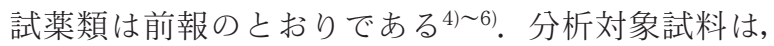
1970 から 2004 年の間に, 大阪府内にて採取された市販 品の食肉（牛肉, 豚肉, 鶏肉) と 1990 加 2004 年の間 に採取された食肉加工食品（食品原材料表示に従って分類 した. 牛肉加工品: コンビーフ, ローストビーフ, ビーフ ジャーキーなど; 豚肉加工品：八ム，ベーコン，焼き豚な ど; 牛肉と豚肉の混合加工品: 各種ソーセージ, ハンバー グなど；鶏肉加工品：焼き鳥, フライドチキン, チキンナ ゲットなど）で, 大部分は大阪府の行政検査として当研究 所に搬入されたあのである. 残りは, 当所にて独自に購入 した，各試料については部位別の区分は行わなかった。ま た，国内に流通するむのを試料とし，国内産および外国産

\section{の区分は行わなかった。}

\section{2. 分析装置}

分析装置および分析条件は前報のとおりである4) 6). た だし，PCB 分析用の GC-ECD は Varian 社製，2100 型， 1400 型および(株)島津製作所製 GC-7 A を使用した。有 機塩素系農薬分析用 GC-ECD には, 1970 から 1989 年の 間はVarian 社製， 2100 型，1400 型を用い，(a) 2\% silicone OV-17, または (b) 2\% diethylene glycol succinate および $0.5 \%$ リン酸を Gas Chrom Q (100/120 mesh) にコーティングしたものを充填したパックドカラ ムを使用した。 1990 から 2004 年までは, Hewlett Packard 社製 5890A, 5890A series II またはVarian 社製, 3300 型を使用した。キャピラリーカラムは，(a) J \& W 社製 DB-1，内径 $0.25 \mathrm{~mm}$ ，長さ $30 \mathrm{~m}$ ，膜厚 $0.5 \mu \mathrm{m}$, (b) $\mathrm{J} \& \mathrm{~W}$ 社製 DB-1701, 内径 $0.25 \mathrm{~mm}$, 長さ $30 \mathrm{~m}$, 膜厚 $0.25 \mu \mathrm{m}$ を用いた。 
Table 2. Concentration Changes of $\mathrm{PCB}$ and Organochlorine Pesticides in Pork Samples Collected on the Market in Osaka, Japan for 35 Years (1970-2004)

\begin{tabular}{|c|c|c|c|c|c|c|c|c|}
\hline \multirow{2}{*}{\multicolumn{2}{|c|}{ Chemicals }} & \multicolumn{7}{|c|}{ Year } \\
\hline & & '70-'74 & '75-'79 & '80-'84 & '85-'89 & '90-'94 & '95-'99 & '00-'04 \\
\hline \multirow{3}{*}{$\mathrm{PCB}$} & $n$ & NT & $7(7)$ & $15(8)$ & $19(4)$ & $16(2)$ & $27(2)$ & $30(0)$ \\
\hline & $\mathrm{Av} \pm \mathrm{SD}(\mathrm{ppb})$ & & $3.9 \pm 2.8^{*}$ & $0.73 \pm 0.80^{\dagger}$ & $0.47 \pm 1.2^{*}$ & $0.13 \pm 0.34^{*}$ & $0.07 \pm 0.27^{* \dagger}$ & $n d^{* \dagger}$ \\
\hline & Range (ppb) & & $1-7$ & nd-2 & nd -5 & nd-1 & nd-2 & nd \\
\hline \multirow{3}{*}{$\mathrm{HCH}^{\mathrm{a})}$} & $n$ & $33(31)$ & $24(24)$ & $15(11)$ & $19(11)$ & $20(13)$ & $39(23)$ & $30(7)$ \\
\hline & $\mathrm{Av} \pm \mathrm{SD}(\mathrm{ppb})$ & $362 \pm 411^{*}$ & $29 \pm 21^{* \dagger}$ & $6.1 \pm 7.3^{* \dagger \#}$ & $2.4 \pm 2.6^{* \dagger}$ & $1.6 \pm 1.6^{* \dagger}$ & $1.9 \pm 2.5^{* \dagger \S}$ & $0.50 \pm 1.0^{* \dagger \# \S ~}$ \\
\hline & Range (ppb) & nd -1622 & $3-82$ & nd -26 & nd -7 & nd -6 & nd -11 & nd -4 \\
\hline \multirow{3}{*}{$\mathrm{DDT}^{\mathrm{b})}$} & $n$ & $33(29)$ & $24(24)$ & $15(14)$ & $19(19)$ & $20(11)$ & $39(36)$ & $30(27)$ \\
\hline & $\mathrm{Av} \pm \mathrm{SD}(\mathrm{ppb})$ & $333 \pm 266^{*}$ & $178 \pm 223^{\dagger}$ & $16 \pm 20^{* \dagger \#}$ & $5.1 \pm 5.0^{* \dagger 8}$ & $1.8 \pm 2.1^{* \dagger \# \S ~}$ & $3.1 \pm 2.8^{* \dagger \#}$ & $2.0 \pm 1.8^{* \dagger \# \S}$ \\
\hline & Range (ppb) & nd-1187 & $1-809$ & nd-68 & $1-7$ & nd-7 & nd-15 & nd-8 \\
\hline \multirow{3}{*}{ Dieldrin } & $n$ & $33(8)$ & $24(17)$ & $15(7)$ & $19(0)$ & $20(0)$ & $39(9)$ & $30(7)$ \\
\hline & $\mathrm{Av} \pm \mathrm{SD}(\mathrm{ppb})$ & $14 \pm 43$ & $4.3 \pm 5.3^{*}$ & $2.7 \pm 5.3^{\dagger}$ & $\mathrm{nd}^{* \dagger}$ & $\mathrm{nd}^{* \dagger}$ & $0.36 \pm 0.71^{*}$ & $0.27 \pm 0.52 *$ \\
\hline & Range (ppb) & nd-240 & nd -24 & nd-16 & nd & nd & nd-2 & nd-2 \\
\hline \multirow{3}{*}{$\mathrm{HCE}^{\mathrm{c})}$} & $n$ & NT & $24(0)$ & $15(0)$ & $19(1)$ & $20(0)$ & $39(3)$ & $30(2)$ \\
\hline & $\mathrm{Av} \pm \mathrm{SD}(\mathrm{ppb})$ & & nd & nd & $0.16 \pm 0.69$ & nd & $0.08 \pm 0.27$ & $0.07 \pm 0.25$ \\
\hline & Range (ppb) & & nd & nd & nd-3 & nd & nd-1 & nd-1 \\
\hline \multirow{3}{*}{$\mathrm{HCB}^{\mathrm{d})}$} & $n$ & NT & $24(3)$ & $15(0)$ & $19(2)$ & $20(0)$ & $39(0)$ & $30(0)$ \\
\hline & $\mathrm{Av} \pm \mathrm{SD}(\mathrm{ppb})$ & & $0.42 \pm 0.12$ & nd & $0.16 \pm 0.50$ & nd & nd & nd \\
\hline & Range (ppb) & & nd -4 & nd & nd -2 & nd & nd & nd \\
\hline
\end{tabular}

The concentration of each chemical (PCB, on a whole basis; each pesticide, on a fat basis) is shown as the average (Av) \pm standard deviation (SD). $n$ : number of samples; those in which chemicals were detected are shown in parentheses. NT: not tested. nd: not detected $(<1 \mathrm{ppb})$; the value was statistically treated as zero. Aldrin and endrin were not detected in any

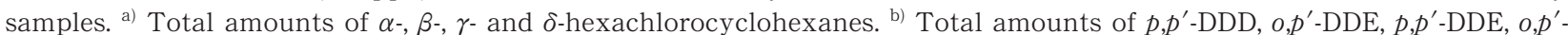
DDT and $p, p^{\prime}$-DDT. ${ }^{c)}$ Total amounts of heptachlor and heptachlor epoxide. ${ }^{\text {d) }}$ Hexachlorobenzene.

$*,+, \#, s$ : Of the values marked by the same symbols, the one in the leftmost column is significantly different from the others $(p<0.05)$.

\section{3. 分析方法}

PCB および有機塩素系農薬の抽出・精製は, 衛生試験 法・注解 ${ }^{21)}$ に準じて行った。概略は以下のと扔りである. PCB 分析では，まず試料に水酸化カリウムを添加後，加 熱還流してアルカリ分解を行った。処理溶液を $n$-ヘキサ ンで抽出し，脱水・濃縮後，フロリジルカラムクロマトグ ラフィー処理を行って PCB を精製・回収した。試料中の PCB 濃度は, 上記装置を用いて鵜川らの PCB 数值化法 ${ }^{13)}$ により全量中濃度として求めた。

有機塩素系農薬の分析では，まず試料をアセトン・ $n$-へ キサン等量混液で混合抽出し, 得られた脂肪に $n$-へキサ ンと $n$-ヘキサン飽和アセトニトリルによる液-液分配処理 を行ってアセトニトリル層に農薬を回収した（3 回）。こ れを $n$-ヘキサンに転溶した後, 濃縮し, さらに, フロリ ジルカラムクロマトグラフィー処理を行って農薬を精製・ 回収した。 GC 分析では, 標準液検量線之試料検液のピー ク高を比較することにより試料中の各種農薬濃度を算定し た. 各種農薬の濃度は, $\mathrm{a}, \mathrm{b}$ 両方のカラムによる值の平均
值を脂肪中濃度として表した。

\section{4. データの統計処理}

得られたデー夕の各年度の化合物濃度比較は, 多重検定 としてすべての群間で対比較を検定する Steel-Dwass の 方法（両側検定）を用いた。また，食肉之食肉加工食品中 の化合物濃度比較では, 対応のない 2 群の比較検定であ る Mann-Whitney's U test（両側検定）を用いた。

\section{結果および考察}

\section{1. 食肉中の PCB および有機塩素系農薬の濃度推移}

食肉（牛肉, 豚肉, 鶏肉) 中の $\mathrm{PCB}$ や有機塩素系農薬 の污染状況の調査では, 年度ごとの調査個数や污染濃度の 多少による標本のばらつきを少なくするため 5 年ごとの 濃度推移を比較検討した。

牛肉中の PCB 検出値は, 70 年代後半から 2000 年代前 半にかけて漸次減少の傾向が観察された (Table 1). HCHs の検出值は，70 年代前半に特に高い值が観察され，この 理由として，HCHs が散布された麦わらを牛の飰に用いた 
Table 3. Concentration Changes of PCB and Organochlorine Pesticides in Poultry Samples Collected on the Market in Osaka, Japan for 35 Years (1970-2004)

\begin{tabular}{|c|c|c|c|c|c|c|c|c|}
\hline \multirow{2}{*}{\multicolumn{2}{|c|}{ Chemicals }} & \multicolumn{7}{|c|}{ Year } \\
\hline & & '70-'74 & '75-'79 & '80-'84 & '85-'89 & '90-'94 & '95-'99 & '00-'04 \\
\hline \multirow{3}{*}{ PCB } & $n$ & NT & $6(6)$ & $14(9)$ & $19(0)$ & $19(4)$ & $23(4)$ & $20(3)$ \\
\hline & $\mathrm{Av} \pm \mathrm{SD}(\mathrm{ppb})$ & & $3.7 \pm 1.8^{*}$ & $2.0 \pm 2.1^{\dagger}$ & $\mathrm{nd}^{* \dagger}$ & $0.21 \pm 0.42^{* \dagger}$ & $0.65 \pm 1.8^{*}$ & $0.20 \pm 0.52^{* \dagger}$ \\
\hline & Range (ppb) & & $1-6$ & nd-6 & nd & nd-1 & nd-7 & nd-2 \\
\hline \multirow{3}{*}{$\mathrm{HCH}^{\mathrm{a})}$} & $n$ & $35(35)$ & $22(22)$ & $14(7)$ & $19(10)$ & $49(36)$ & $24(19)$ & $20(4)$ \\
\hline & $\mathrm{Av} \pm \mathrm{SD}(\mathrm{ppb})$ & $415 \pm 269 *$ & $73 \pm 51^{* \dagger}$ & $14 \pm 19^{* \dagger}$ & $3.5 \pm 5.6^{* \dagger}$ & $3.4 \pm 4.8^{* \dagger \#}$ & $2.5 \pm 2.7^{* \dagger \S}$ & $0.70 \pm 1.9 * \dagger \# \S$ \\
\hline & Range (ppb) & $9-1353$ & $20-196$ & nd-58 & nd-23 & nd-27 & nd-10 & nd-8 \\
\hline \multirow{3}{*}{$\mathrm{DDT}^{\mathrm{b})}$} & $n$ & $35(31)$ & $22(22)$ & $14(14)$ & $19(19)$ & $49(45)$ & $24(23)$ & $20(18)$ \\
\hline & $\mathrm{Av} \pm \mathrm{SD}(\mathrm{ppb})$ & $507 \pm 380 *$ & $306 \pm 327^{\dagger}$ & $26 \pm 14^{* \dagger \#}$ & $51 \pm 7.9 * \dagger s$ & $14 \pm 46^{* \dagger \#}$ & $6.1 \pm 7.5^{* \dagger \#}$ & $3.1 \pm 2.2^{* \dagger} \AA$ \\
\hline & Range (ppb) & nd-1420 & $14-1194$ & $9-58$ & $2-251$ & nd-324 & nd-32 & nd-9 \\
\hline \multirow{3}{*}{ Dieldrin } & $n$ & $35(21)$ & $22(22)$ & $14(6)$ & $19(5)$ & $49(19)$ & $24(16)$ & $20(9)$ \\
\hline & $\mathrm{Av} \pm \mathrm{SD}(\mathrm{ppb})$ & $25 \pm 26^{*}$ & $24 \pm 14^{\dagger}$ & $3.4 \pm 4.7^{\dagger}$ & $1.9 \pm 4.9 *^{\dagger}$ & $1.5 \pm 3.2^{* \dagger}$ & $1.1 \pm 1.4^{\dagger}$ & $0.45 \pm 0.51^{\dagger}$ \\
\hline & Range (ppb) & nd-108 & $4-57$ & nd-14 & nd-21 & nd-19 & $\mathrm{nd}-7$ & nd-1 \\
\hline \multirow{3}{*}{$\mathrm{HCE}^{\mathrm{c})}$} & $n$ & NT & $22(0)$ & $14(0)$ & $19(4)$ & $49(0)$ & $24(1)$ & $20(0)$ \\
\hline & $\mathrm{Av} \pm \mathrm{SD}(\mathrm{ppb})$ & & nd & nd & $0.32 \pm 0.67^{*}$ & $\mathrm{nd}^{*}$ & $0.17 \pm 0.82$ & nd \\
\hline & Range (ppb) & & nd & nd & nd -6 & nd & nd-4 & nd \\
\hline \multirow{3}{*}{$\mathrm{HCB}^{\mathrm{d})}$} & $n$ & NT & $22(3)$ & $14(0)$ & $19(3)$ & $49(0)$ & $24(2)$ & $20(0)$ \\
\hline & $\mathrm{Av} \pm \mathrm{SD}(\mathrm{ppb})$ & & $0.59 \pm 1.6$ & nd & $0.21 \pm 0.54$ & nd & $0.08 \pm 0.28$ & nd \\
\hline & Range (ppb) & & $\mathrm{nd}-5$ & nd & nd-2 & nd & $\mathrm{nd}-1$ & nd \\
\hline
\end{tabular}

The concentration of each chemical (PCB, on a whole basis; each pesticide, on a fat basis) is shown as the average (Av) \pm standard deviation (SD). $n$ : number of samples; those in which chemicals were detected are shown in parentheses. NT: not tested. nd: not detected $(<1 \mathrm{ppb})$; the value was statistically treated as zero. Aldrin and endrin were not detected in any samples. ${ }^{\text {a) }}$ Total amounts of $\alpha-, \beta$-, $\gamma$ - and $\delta$-hexachlorocyclohexanes. ${ }^{\text {b) }}$ Total amounts of $p, p^{\prime}$-DDD, $o, p^{\prime}$-DDE, $p, p^{\prime}$-DDE, $o, p^{\prime}$ DDT and $p, p^{\prime}$-DDT. ${ }^{c)}$ Total amounts of heptachlor and heptachlor epoxide. ${ }^{\text {d) }}$ Hexachlorobenzene.

$*,+, \#, s$ : Of the values marked by the same symbols, the one in the leftmost column is significantly different from the others $(p<0.05)$.

ためと考えられる ${ }^{14)}$. HCHs の使用禁止により ${ }^{2)}, 70$ 年代 前半から後半にかけて急激な減少 (約 $1 / 31$ ) が観察され たが，以降では減少の幅は少なく，2000 年代前半では $\mathrm{ppb}$ オーダーの平均值を示した。 DDTs の検出平均值は, 70 年代前半から 80 年代前半の 10 年間で 5 年間ごとに約 1/6の減少となったが，それ以降は ppbオーダーの值を 示した。 また, dieldrin では, 70 年代前半から 80 年代 前半にかけて減少傾向（約 1/2.2）が観察されたが, 以降 は低濃度の残留持続が観察された。. Aldrin および endrin は全調査期間で検出されなかった. HCE (heptachlor は 検出されず）や HCBでは, 全調査期間を通して残留濃度 は低值を示した。

豚肉中の $\mathrm{PCB}, \mathrm{HCH}$, DDTs, dieldrin の検出值は, 調 查期間中に漸次減少の傾向を示した (Table 2). HCHs の 検出平均值は, 70 年代前半から後半にかけて約 $1 / 12$ の 減少であり, 牛肉に比べ比較的緩和な濃度減少と言える. DDTs の検出平均值は, 70 年代前半から後半にかけて濃 度減衰の割合は小さく（約 $1 / 1.9$, 以下の Table 3 のと
おり鶏肉でも約 1/1.7)，70 年代後半から 80 年代前半で は約 1/11（Table 3 のとおり鶏肉でも約 1/12）を示し

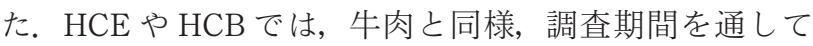
残留濃度は低值を示した。

鶏肉においても PCB, HCHs, DDTs, dieldrin の検出値 は，調査期間中において漸次減少傾向が観察された (Table 3). HCHs の検出平均值は 70 年代前半から後半に かけて比較的緩やかな減少（約 1/5.7）となり，豚肉之類 似の傾向が観察された。 HCE や HCB では, 牛肉, 豚肉 と同様, 調査期間を通して残留濃度は低值を示した。

\section{2. 食肉加工食品中の有機塩素系農薬の残留濃度}

15 年間（1990 から 2004 年）の各食肉別による加工食 品中農薬の濃度を比較したところ, 牛肉, 豚肉, 鶏肉, お よび牛と豚の混合加工食品中の平均濃度は, 各農薬ともに 大きな相違は認められなかった (Table 4). 牛肉加工食品 の $\mathrm{HCHs}$ 平均值は, 同一期間における牛肉中の平均値 （7.4 ppb，集計データは示さず）に比べ約 $1 / 10$ の低い值 （有意差あり）となった。 DDTs の平均值では，同一期間 
Table 4. Concentration of Organochlorine Pesticides in Processed Meat Products Collected on the Market in Osaka, Japan for 15 Years (1990-2004)

\begin{tabular}{|c|c|c|c|c|c|}
\hline \multirow{2}{*}{\multicolumn{2}{|c|}{ Chemicals }} & \multicolumn{4}{|c|}{ Items } \\
\hline & & Processed beef & Processed pork & Mixture $^{\text {a) }}$ & Processed poultry \\
\hline \multirow{3}{*}{$\mathrm{HCH}^{\mathrm{b})}$} & $n$ & $34(9)$ & $63(18)$ & $38(19)$ & $9(4)$ \\
\hline & $\mathrm{Av} \pm \mathrm{SD}(\mathrm{ppb})$ & $0.71 \pm 1.6$ & $1.0 \pm 2.5$ & $1.4 \pm 2.8$ & $2.3 \pm 3.7$ \\
\hline & Range (ppb) & nd-8 & nd -14 & nd -14 & nd -11 \\
\hline \multirow{3}{*}{$\mathrm{DDT}^{\mathrm{c})}$} & $n$ & $34(26)$ & $63(48)$ & $38(30)$ & $9(6)$ \\
\hline & $\mathrm{Av} \pm \mathrm{SD}(\mathrm{ppb})$ & $2.2 \pm 3.4$ & $2.2 \pm 2.7$ & $2.0 \pm 2.5$ & $2.4 \pm 3.0$ \\
\hline & Range (ppb) & nd -19 & nd -13 & nd -13 & nd -9 \\
\hline \multirow{3}{*}{ Dieldrin } & $n$ & $34(5)$ & $63(7)$ & $38(4)$ & $9(1)$ \\
\hline & $\mathrm{Av} \pm \mathrm{SD}(\mathrm{ppb})$ & $0.15 \pm 0.36$ & $0.19 \pm 0.62$ & $0.18 \pm 0.56$ & $0.11 \pm 0.33$ \\
\hline & Range (ppb) & nd-1 & nd-3 & nd-2 & nd -1 \\
\hline \multirow{3}{*}{$\mathrm{HCE}^{\mathrm{d})}$} & $n$ & $34(1)$ & $63(1)$ & $38(1)$ & $9(0)$ \\
\hline & $\mathrm{Av} \pm \mathrm{SD}(\mathrm{ppb})$ & $0.03 \pm 0.17$ & $0.02 \pm 0.13$ & $0.03 \pm 0.16$ & nd \\
\hline & Range (ppb) & nd-1 & nd-1 & nd-1 & nd \\
\hline \multirow{3}{*}{$\mathrm{HCB}^{\mathrm{e})}$} & $n$ & $34(2)$ & $63(0)$ & $38(5)$ & $9(2)$ \\
\hline & $\mathrm{Av} \pm \mathrm{SD}(\mathrm{ppb})$ & $0.06 \pm 0.24$ & nd & $0.24 \pm 0.71$ & $0.33 \pm 0.71$ \\
\hline & Range (ppb) & $n d-1$ & nd & nd-3 & $n d-2$ \\
\hline
\end{tabular}

The concentration of each chemical (ppb on a fat basis) is shown as the average (Av) \pm standard deviation (SD). $n$ : number of samples; those in which chemicals were detected are shown in parentheses. nd: not detected $(<1 \mathrm{ppb})$; the value was statistically treated as zero. Aldrin and endrin were not detected in any samples. ${ }^{\text {a) }}$ Processed mixture of ground beef

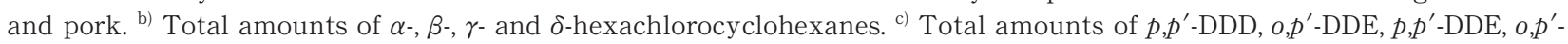
DDT and $p, p^{\prime}$-DDT. ${ }^{\text {d) }}$ Total amounts of heptachlor and heptachlor epoxide. ${ }^{\text {e) }}$ Hexachlorobenzene.

の牛肉 (4.2 ppb, 集計データは示さず) と比べて加工食 品は約 $1 / 2$ の值（有意差なし）となった。 また, dieldrin, $\mathrm{HCE}, \mathrm{HCB}$ の平均值も牛肉に比べ加工食品のほうが 低い傾向が得られた。豚肉とその加工食品との間で各種農 薬の濃度比較を行ったところ, 加工食品のほうがやや低い 傾向を示した（集計デー夕は示さず）。また，䳕肉とその 加工品の濃度比較では, HCHs は同程度であったが, DDTs では加工品のほうが低い傾向が観察された。

\section{3. 食肉中の $\mathrm{HCH}$ 各異性体の濃度推移}

Table 5 では, 食肉中の $\mathrm{HCH}$ 各異性体の濃度推移を調 ベたところ，牛肉では，それぞれ全調査期間を通して漸次 減少傾向が観察された。 また, どの観察年度も $\beta$-体の濃 度が最む高く, 次いで $\alpha-, \gamma-, \delta$-体の順であった. $\mathrm{HCH}$ 工 業品の組成例は， $\alpha$-体 55～80\%， $\beta$-体 5〜14\%， $\gamma$-体 8〜 $15 \% ， \delta$-体 $2 \sim 16 \%$ と報告されている ${ }^{15)}$ が， $\alpha$-, $\gamma$-, $\delta$-体の 牛体内代謝が速いため, $\beta$-体の残留が主体となったものと 考えられる。

豚肉および鶏肉では, 牛肉と同様, 各異性体の検出值は それぞれ調査期間を通して漸次減少が観察された (Table 5). また, どの調査年度む $\beta$-体の濃度が最も高く（80 年 代前半では $\alpha$-体 $>\beta$-体), 次いで $\alpha$-, $\gamma$-, $\delta$-体の順であった.

また, 食肉加工食品中の $\mathrm{HCH}$ 各異性体の濃度比較を 行ったところ, どの加工食品においても $\beta$-体の濃度が最
屯高く，次いで $\alpha$-, $\gamma$-, $\delta$-体の順に高い濃度傾向を示した (デー夕は示さず).

\section{4. 食肉中の DDT 関連化合物の濃度推移}

食肉中の DDT 関連化合物の濃度推移を調べたところ, 牛肉, 豚肉, 鶏肉のいずれも, 主に $p, p^{\prime}-\mathrm{DDD}, p, p^{\prime}-\mathrm{DDE}$, $p, p^{\prime}$-DDT の污染が観察され, その污染は年代経過ととも に減少の傾向を示した (Table 6)。 $o, p^{\prime}$-DDE, $o, p^{\prime}$-DDT は，各食肉で全調査期間を通してそれぞれわずかに検出が 認められたのみであった。 DDT工業品の組成例 ${ }^{16)}$ は, p,p'-DDD: 0.17〜4.0\%,o, $p^{\prime}$-DDD: 0.04\%,o, $p^{\prime}$-DDT: $19 \sim$ $21 \%, p, p^{\prime}$-DDT: $65 \sim 73 \%$ との報告があるが，70 年代前 半の牛肉では, 各化合物が牛体内代謝を受けたため ${ }^{17)}$, 代 謝物 $p, p^{\prime}-\mathrm{DDD}(22 \%)$ や $p, p^{\prime}-\mathrm{DDE}(42 \%)$ ，および原体 の $p, p^{\prime}$-DDT (36\%) の割合が高い. また， 70 年代後半か ら 2000 年前半にかけて $p, p^{\prime}-\mathrm{DDD}, p, p^{\prime}$-DDT が代謝を受 けて ${ }^{17)}$ それらの割合が低くなるにつれ，代謝物 $p, p^{\prime}-\mathrm{DDE}$ の割合が高くなる傾向が認められた。豚肉や鶏肉において 屯牛肉と同様の濃度推移が得られたが，鶏肉では 80 年代 後半から 90 年代前半にかけては $p, p^{\prime}$-DDT の比較的高濃 度の試料が検出されたため, $p, p^{\prime}$-DDT の割合が高く推移 した。 また, 食肉加工食品中の DDTs の濃度比較を行っ たところ，どの加工食品においても， $p, p^{\prime}-\mathrm{DDE}$ の濃度が 高く, 次いで $p, p^{\prime}$-DDT の濃度が高い傾向が観察された 
Table 5. Concentration Changes of 4 Isomers of Hexachlorocyclohexane (HCHs) in Meats Collected on the Market in Osaka, Japan for 35 Years (1970-2004)

\begin{tabular}{|c|c|c|c|c|c|c|c|c|c|}
\hline \multirow{2}{*}{ Items } & \multirow{2}{*}{\multicolumn{2}{|c|}{ Chemicals }} & \multicolumn{7}{|c|}{ Year } \\
\hline & & & '70-'74 & $' 75-' 79$ & '80-'84 & '85-'89 & '90-'94 & '95-’99 & '00-'04 \\
\hline \multirow{12}{*}{ Beef } & \multirow{3}{*}{$\alpha-\mathrm{HCH}$} & $n$ & $47(45)$ & $26(26)$ & $18(15)$ & $42(14)$ & $42(11)$ & $40(12)$ & $25(2)$ \\
\hline & & $\mathrm{Av} \pm \mathrm{SD}(\mathrm{ppb})$ & $427 \pm 997^{*}$ & $22 \pm 13^{* \dagger}$ & $8.9 \pm 7.3^{* \dagger \#}$ & $1.4 \pm 3.4^{* \dagger \#}$ & $1.8 \pm 6.1^{* \dagger \#}$ & $0.65 \pm 1.4^{* \dagger} \#$ & $0.16 \pm 0.62^{* \dagger \#}$ \\
\hline & & Range (ppb) & nd-6667 & $3-50$ & nd-25 & nd-18 & nd-38 & $n d-6$ & $n d-3$ \\
\hline & \multirow{3}{*}{$\beta-\mathrm{HCH}$} & $n$ & $47(46)$ & $26(26)$ & $18(12)$ & $42(17)$ & $42(27)$ & $40(22)$ & $25(5)$ \\
\hline & & $\mathrm{Av} \pm \mathrm{SD}(\mathrm{ppb})$ & $1986 \pm 3418^{*}$ & $51 \pm 40^{* \dagger}$ & $13 \pm 16^{* \dagger \#}$ & $3.9 \pm 12^{* \dagger \#}$ & $9.3 \pm 27^{* \dagger}$ & $4.3 \pm 10^{* \dagger}$ & $4.1 \pm 19^{* \dagger \#}$ \\
\hline & & Range (ppb) & nd-16410 & $5-128$ & nd-64 & nd-74 & nd-157 & $n d-46$ & nd-96 \\
\hline & \multirow{3}{*}{$\gamma-\mathrm{HCH}$} & $n$ & $47(39)$ & $26(22)$ & $18(10)$ & $42(4)$ & $42(3)$ & $40(1)$ & $25(0)$ \\
\hline & & $\mathrm{Av} \pm \mathrm{SD}(\mathrm{ppb})$ & $125 \pm 495^{*}$ & $8.3 \pm 7.1^{* \dagger}$ & $1.6 \pm 2.1^{* \dagger \#}$ & $0.17 \pm 0.58^{* \dagger \#}$ & $0.26 \pm 1.2^{* \dagger \#}$ & $0.05 \pm 0.32^{* \dagger}$ & $\mathrm{nd}^{* \dagger} \#$ \\
\hline & & Range (ppb) & nd-3400 & nd-28 & nd-8 & nd-3 & $n d-7$ & nd-2 & nd \\
\hline & \multirow{3}{*}{$\delta-\mathrm{HCH}$} & $n$ & $47(33)$ & $26(14)$ & $18(0)$ & $42(0)$ & $42(1)$ & $40(0)$ & $25(0)$ \\
\hline & & $\mathrm{Av} \pm \mathrm{SD}(\mathrm{ppb})$ & $55 \pm 134^{*}$ & $2.8 \pm 4.1 *^{\dagger}$ & $\mathrm{nd}^{* \dagger}$ & $\mathrm{nd}^{* \dagger}$ & $0.02 \pm 0.15^{* \dagger}$ & $\mathrm{nd}^{* \dagger}$ & $\mathrm{nd}^{* \dagger}$ \\
\hline & & Range (ppb) & nd-853 & nd-18 & nd & nd & nd-1 & nd & nd \\
\hline \multirow{12}{*}{ Pork } & \multirow{3}{*}{$\alpha-\mathrm{HCH}$} & $n$ & $33(28)$ & $24(23)$ & $15(10)$ & $19(5)$ & $20(4)$ & $39(6)$ & $30(2)$ \\
\hline & & $\mathrm{Av} \pm \mathrm{SD}(\mathrm{ppb})$ & $75 \pm 72^{*}$ & $10 \pm 7.4^{* \dagger}$ & $3.6 \pm 4.1^{* \dagger \#}$ & $0.37 \pm 0.68^{* \dagger}$ & $0.35 \pm 0.75^{* \dagger} \#$ & $0.23 \pm 0.58^{* \dagger} \#$ & $0.07 \pm 0.25 * \dagger$ \\
\hline & & Range (ppb) & nd-240 & nd-28 & nd-12 & $\mathrm{nd}-2$ & $n d-2$ & $n d-2$ & $\mathrm{nd}-1$ \\
\hline & \multirow{3}{*}{$\beta-\mathrm{HCH}$} & $n$ & $33(25)$ & $24(22)$ & $15(6)$ & $19(10)$ & $20(13)$ & $39(21)$ & $30(6)$ \\
\hline & & $\mathrm{Av} \pm \mathrm{SD}(\mathrm{ppb})$ & $260 \pm 334^{*}$ & $14 \pm 16^{* \dagger}$ & $2.1 \pm 3.4^{* \dagger}$ & $2.0 \pm 2.3^{* \dagger}$ & $1.3 \pm 1.5^{* \dagger}$ & $1.6 \pm 2.3^{* \dagger}$ & $0.27 \pm 0.58^{* \dagger}$ \\
\hline & & Range (ppb) & nd-1383 & nd-64 & nd -12 & $\mathrm{nd}-7$ & $n d-6$ & nd-11 & $\mathrm{nd}-2$ \\
\hline & \multirow{3}{*}{$\gamma-\mathrm{HCH}$} & $n$ & $33(20)$ & $24(20)$ & $15(3)$ & $19(1)$ & $20(0)$ & $39(2)$ & $30(3)$ \\
\hline & & $\mathrm{Av} \pm \mathrm{SD}(\mathrm{ppb})$ & $12 \pm 20^{*}$ & $3.8 \pm 4.7^{\dagger}$ & $0.47 \pm 1.1^{* \dagger}$ & $0.05 \pm 23^{\dagger \dagger}$ & $\mathrm{nd}^{* \dagger}$ & $0.08 \pm 0.35^{* \dagger}$ & $0.17 \pm 0.53^{* \dagger}$ \\
\hline & & Range (ppb) & nd-100 & nd-22 & $n d-4$ & nd-1 & nd & nd-2 & $\mathrm{nd}-2$ \\
\hline & \multirow{3}{*}{$\delta$-HCH } & $n$ & $33(17)$ & $24(7)$ & $15(0)$ & $19(0)$ & $20(0)$ & $39(0)$ & $30(0)$ \\
\hline & & $\mathrm{Av} \pm \mathrm{SD}(\mathrm{ppb})$ & $15 \pm 29 *$ & $1.0 \pm 1.9^{\dagger}$ & $\mathrm{nd}^{*}$ & $\mathrm{nd}^{*}$ & $\mathrm{nd}^{*}$ & $\mathrm{nd}^{* \dagger}$ & $\mathrm{nd}^{* \dagger}$ \\
\hline & & Range (ppb) & nd-133 & nd-6 & nd & nd & nd & nd & nd \\
\hline \multirow{12}{*}{ Poultry } & \multirow{3}{*}{$\alpha-\mathrm{HCH}$} & $n$ & $35(33)$ & $22(22)$ & $14(7)$ & $19(3)$ & $49(13)$ & $24(6)$ & $20(1)$ \\
\hline & & $\mathrm{Av} \pm \mathrm{SD}(\mathrm{ppb})$ & $88 \pm 132^{*}$ & $29 \pm 20 * \dagger$ & $10 \pm 14^{* \dagger \#}$ & $0.74 \pm 2.7^{* \dagger}$ & $0.61 \pm 1.2^{* \dagger}$ & $0.58 \pm 1.3^{* \dagger}$ & $0.15 \pm 0.67 * \dagger \#$ \\
\hline & & Range (ppb) & nd-800 & $3-66$ & nd-43 & nd-12 & $n d-4$ & $\mathrm{nd}-5$ & nd-3 \\
\hline & \multirow{3}{*}{$\beta-\mathrm{HCH}$} & $n$ & $35(33)$ & $22(20)$ & $14(3)$ & $19(10)$ & $49(33)$ & $24(17)$ & $20(4)$ \\
\hline & & $\mathrm{Av} \pm \mathrm{SD}(\mathrm{ppb})$ & $275 \pm 178^{*}$ & $31 \pm 27^{* \dagger}$ & $2.6 \pm 7.0^{* \dagger}$ & $2.5 \pm 2.9^{* \dagger}$ & $1.9 \pm 1.9 * \dagger \#$ & $1.5 \pm 1.5 * \dagger \S$ & $0.55 \pm 1.4^{* \dagger \# \S}$ \\
\hline & & Range (ppb) & nd-640 & nd-100 & nd-26 & nd-9 & nd-7 & $\mathrm{nd}-6$ & $\mathrm{nd}-5$ \\
\hline & \multirow{3}{*}{$\gamma$-HCH } & $n$ & $35(33)$ & $22(19)$ & $14(4)$ & $19(3)$ & $49(5)$ & $24(4)$ & $20(0)$ \\
\hline & & $\mathrm{Av} \pm \mathrm{SD}(\mathrm{ppb})$ & $41 \pm 26^{*}$ & $9.5 \pm 7.8^{* \dagger}$ & $1.4 \pm 2.4^{* \dagger}$ & $0.32 \pm 0.94^{* \dagger}$ & $0.84 \pm 3.3^{* \dagger}$ & $0.29 \pm 0.86^{* \dagger}$ & $\mathrm{nd}^{* \dagger}$ \\
\hline & & Range (ppb) & nd-100 & nd-24 & $\mathrm{nd}-7$ & nd-4 & nd-21 & $\mathrm{nd}-4$ & nd \\
\hline & \multirow{3}{*}{$\delta-\mathrm{HCH}$} & $n$ & $35(16)$ & $22(10)$ & $14(0)$ & $19(0)$ & $49(0)$ & $24(1)$ & $20(0)$ \\
\hline & & $\mathrm{Av} \pm \mathrm{SD}(\mathrm{ppb})$ & $11 \pm 14^{*}$ & $2.9 \pm 4.8^{\dagger}$ & $\mathrm{nd}^{*}$ & $\mathrm{nd}^{* \dagger}$ & $\mathrm{nd}^{* \dagger}$ & $0.04 \pm 0.20^{* \dagger}$ & $\mathrm{nd}^{* \dagger}$ \\
\hline & & Range (ppb) & nd-40 & nd-14 & nd & nd & nd & nd-1 & nd \\
\hline
\end{tabular}

The concentration of each chemical (ppb on a fat basis) is shown as the average (Av) \pm standard deviation (SD). $n$ : number of samples; those in which chemicals were detected are shown in parentheses. nd: not detected $(<1 \mathrm{ppb})$; the value was statistically treated as zero.

$*, \dagger, \#, \S$ : Of the values marked by the same symbols, the one in the leftmost column is significantly different from the others $(p<0.05)$. 
Table 6. Concentration Changes of DDT Related Compounds in Meats Collected on the Market in Osaka, Japan for 35 Years (1970-2004)

\begin{tabular}{|c|c|c|c|c|c|c|c|c|c|}
\hline \multirow{2}{*}{ Items } & \multirow{2}{*}{\multicolumn{2}{|c|}{ Chemicals }} & \multicolumn{7}{|c|}{ Year } \\
\hline & & & '70-'74 & $' 75-' 79$ & '80-'84 & '85-'89 & '90-'94 & '95-'99 & '00-'04 \\
\hline \multirow{15}{*}{ Beef } & \multirow{3}{*}{$p, p^{\prime}-\mathrm{DDD}$} & $n$ & $47(12)$ & $26(11)$ & $18(1)$ & $42(1)$ & $42(1)$ & $40(0)$ & $25(1)$ \\
\hline & & $\mathrm{Av} \pm \mathrm{SD}(\mathrm{ppb})$ & $65 \pm 155^{*}$ & $4.5 \pm 1.1^{\dagger}$ & $0.11 \pm 0.47$ & $0.17 \pm 1.1^{* \dagger}$ & $0.05 \pm 0.31^{* \dagger}$ & $\mathrm{nd}^{* \dagger}$ & $0.04 \pm 0.20^{\dagger}$ \\
\hline & & Range (ppb) & $\mathrm{nd}-665$ & nd-56 & $\mathrm{nd}-2$ & nd-7 & nd-2 & nd & nd-1 \\
\hline & \multirow{3}{*}{$o, p^{\prime}-\mathrm{DDE}$} & $n$ & NT & $26(0)$ & $18(0)$ & $42(0)$ & $42(0)$ & $40(0)$ & $25(2)$ \\
\hline & & $\mathrm{Av} \pm \mathrm{SD}(\mathrm{ppb})$ & & nd & nd & nd & nd & nd & $0.08 \pm 0.28$ \\
\hline & & Range (ppb) & & nd & nd & nd & nd & nd & nd-1 \\
\hline & \multirow{3}{*}{$p, p^{\prime}-\mathrm{DDE}$} & $n$ & $47(40)$ & $26(26)$ & $18(13)$ & $42(32)$ & $42(21)$ & $40(39)$ & $25(15)$ \\
\hline & & $\mathrm{Av} \pm \mathrm{SD}(\mathrm{ppb})$ & $121 \pm 141^{*}$ & $33 \pm 42^{* \dagger}$ & $5.9 \pm 6.6^{* \dagger}$ & $6.3 \pm 9.2^{* \dagger \#}$ & $5.7 \pm 18^{* \dagger \#}$ & $2.8 \pm 4.1^{* \dagger}$ & $3.1 \pm 7.2^{* \dagger}$ \\
\hline & & Range (ppb) & nd-834 & $1-221$ & nd-22 & nd -50 & nd-107 & nd-26 & nd-36 \\
\hline & \multirow{3}{*}{$o, p^{\prime}-\mathrm{DDT}$} & $n$ & $47(0)$ & $26(0)$ & $18(0)$ & $42(0)$ & $42(0)$ & $40(1)$ & $25(0)$ \\
\hline & & $\mathrm{Av} \pm \mathrm{SD}(\mathrm{ppb})$ & nd & nd & nd & nd & nd & $0.03 \pm 0.16$ & nd \\
\hline & & Range (ppb) & nd & nd & nd & nd & nd & nd-1 & nd \\
\hline & \multirow{3}{*}{$p, p^{\prime}-\mathrm{DDT}$} & $n$ & $47(27)$ & $26(15)$ & $18(5)$ & $42(8)$ & $42(2)$ & $40(2)$ & $25(1)$ \\
\hline & & $\mathrm{Av} \pm \mathrm{SD}(\mathrm{ppb})$ & $104 \pm 153^{*}$ & $11 \pm 16^{\dagger}$ & $1.6 \pm 3.0 *$ & $0.88 \pm 2.1^{* \dagger}$ & $0.31 \pm 1.9^{* \dagger}$ & $0.08 \pm 0.34^{* \dagger}$ & $0.04 \pm 0.20^{* \dagger}$ \\
\hline & & Range (ppb) & nd-746 & nd-70 & nd -10 & nd-9 & nd-12 & $\mathrm{nd}-2$ & nd-1 \\
\hline \multirow{15}{*}{ Pork } & \multirow{3}{*}{$p, p^{\prime}-\mathrm{DDD}$} & $n$ & $33(11)$ & $24(17)$ & $15(0)$ & $19(0)$ & $20(0)$ & $39(0)$ & $30(0)$ \\
\hline & & $\mathrm{Av} \pm \mathrm{SD}(\mathrm{ppb})$ & $35 \pm 63^{*}$ & $8.0 \pm 7.9^{\dagger}$ & $\mathrm{nd}^{\dagger}$ & $\mathrm{nd}^{\dagger}$ & $\mathrm{nd}^{\dagger}$ & $\mathrm{nd}^{* \dagger}$ & $\mathrm{nd}^{* \dagger}$ \\
\hline & & Range (ppb) & nd-204 & nd -25 & nd & nd & nd & nd & nd \\
\hline & \multirow{3}{*}{$o, p^{\prime}-\mathrm{DDE}$} & $n$ & NT & $24(0)$ & $15(0)$ & $19(0)$ & $20(0)$ & $39(2)$ & $30(5)$ \\
\hline & & $\mathrm{Av} \pm \mathrm{SD}(\mathrm{ppb})$ & & nd & nd & nd & nd & $0.13 \pm 0.66$ & $0.17 \pm 0.38$ \\
\hline & & Range (ppb) & & nd & nd & nd & nd & $\mathrm{nd}-4$ & nd-1 \\
\hline & \multirow{3}{*}{$p, p^{\prime}-\mathrm{DDE}$} & $n$ & $33(27)$ & $24(24)$ & $15(11)$ & $19(18)$ & $20(11)$ & $39(35)$ & $30(27)$ \\
\hline & & $\mathrm{Av} \pm \mathrm{SD}(\mathrm{ppb})$ & $101 \pm 105^{*}$ & $61 \pm 70^{\dagger}$ & $11 \pm 15^{* \dagger \#}$ & $4.2 \pm 4.4^{* \dagger \S}$ & $1.6 \pm 1.8^{* \dagger \#}$ & $2.1 \pm 1.9$ *†\# & $1.5 \pm 0.94^{* \dagger \# \S ~}$ \\
\hline & & Range (ppb) & nd -467 & $1-318$ & $n d-60$ & nd-20 & $n d-6$ & $\mathrm{nd}-8$ & $n d-4$ \\
\hline & \multirow{3}{*}{$o, p^{\prime}-\mathrm{DDT}$} & $n$ & $33(0)$ & $24(0)$ & $15(0)$ & $19(0)$ & $20(0)$ & $39(2)$ & $30(0)$ \\
\hline & & $\mathrm{Av} \pm \mathrm{SD}(\mathrm{ppb})$ & nd & nd & nd & nd & nd & $0.05 \pm 0.22$ & nd \\
\hline & & Range (ppb) & nd & nd & nd & nd & nd & nd-1 & nd \\
\hline & \multirow{3}{*}{$p, p^{\prime}-\mathrm{DDT}$} & $n$ & $33(25)$ & $24(21)$ & $15(9)$ & $19(4)$ & $20(1)$ & $39(14)$ & $30(7)$ \\
\hline & & $\mathrm{Av} \pm \mathrm{SD}(\mathrm{ppb})$ & $197 \pm 163^{*}$ & $109 \pm 152^{\dagger}$ & $5.9 \pm 8.3^{* \dagger \#}$ & $0.89 \pm 2.0^{* \dagger}$ & $0.20 \pm 0.89 * \dagger$ & $0.84 \pm 1.6^{* \dagger}$ & $0.37 \pm 0.76^{* \dagger}$ \\
\hline & & Range (ppb) & nd-600 & nd-620 & nd-28 & $n d-6$ & $n d-4$ & nd-7 & $n d-3$ \\
\hline \multirow{6}{*}{ Poultry } & \multirow{3}{*}{$p, p^{\prime}-\mathrm{DDD}$} & $n$ & $35(5)$ & $22(9)$ & $14(3)$ & $19(3)$ & $49(5)$ & $24(2)$ & $20(0)$ \\
\hline & & $\mathrm{Av} \pm \mathrm{SD}(\mathrm{ppb})$ & $23 \pm 87$ & $7.4 \pm 20^{*}$ & $0.57 \pm 1.3$ & $1.1 \pm 2.4$ & $0.92 \pm 3.7^{*}$ & $0.21 \pm 0.72$ & $\mathrm{nd}^{*}$ \\
\hline & & Range (ppb) & nd -487 & nd-92 & $\mathrm{nd}-4$ & nd-8 & nd-22 & nd-3 & nd \\
\hline & \multirow{3}{*}{$o, p^{\prime}-\mathrm{DDE}$} & $n$ & $\mathrm{NT}$ & $22(0)$ & $14(0)$ & $19(0)$ & $49(0)$ & $24(0)$ & $20(0)$ \\
\hline & & $\mathrm{Av} \pm \mathrm{SD}(\mathrm{ppb})$ & & nd & nd & nd & nd & nd & nd \\
\hline & & Range (ppb) & & nd & nd & nd & nd & nd & nd \\
\hline
\end{tabular}


Table 6. continued

\begin{tabular}{|c|c|c|c|c|c|c|c|c|c|}
\hline \multirow{2}{*}{ Items } & \multirow{2}{*}{\multicolumn{2}{|c|}{ Chemicals }} & \multicolumn{7}{|c|}{ Year } \\
\hline & & & '70-'74 & '75-'79 & '80-'84 & '85-'89 & '90-'94 & '95-'99 & '00-'04 \\
\hline \multirow{9}{*}{ Poultry } & \multirow{3}{*}{$p, p^{\prime}-\mathrm{DDE}$} & $n$ & $35(30)$ & $22(22)$ & $14(13)$ & $19(19)$ & $49(45)$ & $24(22)$ & $20(18)$ \\
\hline & & $\mathrm{Av} \pm \mathrm{SD}(\mathrm{ppb})$ & $218 \pm 193^{*}$ & $119 \pm 119^{\dagger}$ & $23 \pm 14^{* \dagger \#}$ & $16 \pm 16^{* \dagger \$}$ & $5.1 \pm 4.9 * \dagger \# \&$ & $3.8 \pm 2.4$ *†\# $\$$ & $3.0 \pm 1.9 * \dagger \#$ \\
\hline & & Range (ppb) & nd -871 & $14-555$ & nd -52 & $2-56$ & nd -21 & nd -12 & nd -7 \\
\hline & \multirow{3}{*}{$o, p^{\prime}-\mathrm{DDT}$} & $n$ & $35(0)$ & $22(0)$ & $14(0)$ & $19(0)$ & $49(0)$ & $24(1)$ & $20(0)$ \\
\hline & & $\mathrm{Av} \pm \mathrm{SD}(\mathrm{ppb})$ & nd & nd & nd & nd & nd & $0.04 \pm 0.20$ & nd \\
\hline & & Range (ppb) & nd & nd & nd & nd & nd & nd & nd \\
\hline & \multirow{3}{*}{$p, p^{\prime}-\mathrm{DDT}$} & $n$ & $35(29)$ & $22(19)$ & $14(5)$ & $19(6)$ & $49(7)$ & $24(11)$ & $20(1)$ \\
\hline & & $\mathrm{Av} \pm \mathrm{SD}(\mathrm{ppb})$ & $266 \pm 232^{*}$ & $180 \pm 229^{\dagger}$ & $2.3 \pm 4.1^{* \dagger}$ & $34 \pm 62^{* \dagger}$ & $7.4 \pm 41^{* \dagger}$ & $2.1 \pm 4.7^{* \dagger}$ & $0.10 \pm 0.44^{* \dagger}$ \\
\hline & & Range (ppb) & nd-990 & nd-800 & nd-14 & nd-192 & nd-284 & nd -17 & nd-2 \\
\hline
\end{tabular}

The concentration of each chemical (ppb on a fat basis) is shown as the average (Av) \pm standard deviation (SD). $n$ : number of samples; those in which chemicals were detected are shown in parentheses. nd: not detected $(<1 \mathrm{ppb})$; the value was statistically treated as zero.

$*, \uparrow, \#, \S$ : Of the values marked by the same symbols, the one in the leftmost column is significantly different from the others $(p<0.05)$.

\section{(データは示さず).}

以上，食肉および食肉加工食品中の PCB や有機塩素系 農薬の残留実態を長期間調査したところ，規制值を超える 濃度の試料は検出されなかったが, HCHs や DDTs が比 較的高濃度に残留している試料が近年でも希に検出される ことがあることが分かった。これらの試料は現在の毒性情 報から考えて特にヒトに問題を及ぼす濃度とは言えない が，一部の合成化学物質による低用量での毒性発現の可能 性 ${ }^{10)}$, 11) や，DDTs や PCB 同族体による悪性腫瘍惹起の懸 念 ${ }^{18)}$, 19) を考慮するならば, 食の安全を確保するためこれ らの污染物質の監視体制を今後む継続していくことが重要 であると考えられる。

\section{謝 辞}

本調査において, 試料の搬入などにご助力を賜りました 大阪府環境保健部の皆様方に深謝いたします。また, 試料 の分析などにご協力いただきました当所食品化学課の皆様 方に御礼申し上げます。

\section{文献}

1) Araki, S. et al. eds., "Kankyo Kagaku Jiten”, Tokyo, Tokyo Kagaku Doujin Co., 1985, p. 749-750.

2) Uemura, S., Kawamura, H., Tsuji, M., Tomita, S., Maeda, S., "Noyaku Dokusei No Jiten Kaiteiban", Tokyo, Sanseido Publ. Co., 2002, p. 121-124, p. 183186, p. 188-190, p. 239-244, p. 264-268.

3) Suzuki, T., Ishizaka, T., Sasaki, K., Saito, Y., Hukuda, H., Pesticide residues in imported Australian meats. Shokuhin Eiseigaku Zasshi (J. Food Hyg. Soc. Japan), 30, 48-53 (1989).

4) Kuwabara, K., Matsumoto, H., Murakami, Y., Nishimune, T., Sueki, K., Tanaka, R., Kashimoto, T., Contam- ination profile of some fish and shellfish by organochlorine compounds. Shokuhin Eiseigaku Zasshi (J. Food Hyg. Soc. Japan), 30, 359-366 (1989).

5) Murakami, Y., Kuwabara, K., Matsumoto, H., Kajimoto, M., Nishimune, T., Sasaki, Y., Analysis of organochlorine pesticides in imported foods. Bull. Osaka Pref. Inst. Publ. Health (Ed. Food Hyg.), 26, 33-43 (1995).

6) Matsumoto, H., Murakami, Y., Kuwabara, K., Murata, H., Kitagawa, M., Imaida, M., Nishimune, T., Sasaki Y., Sueki, K., Survey of residual PCB and pesticides in nutrient-supplementary foods. Shokuhin Eiseigaku Zasshi (J. Food Hyg. Soc. Japan), 37, 123-126 (1996).

7) Furusawa, N., Morita, Y., Polluting profiles of dieldrin and DDTs in laying hens of Osaka, Japan. J. Vet. Med. B Infect. Dis. Vet. Public Health., 47, 511-515 (2000).

8) Matsumoto, H., Murakami, Y., Kuwabara, K., "Analytical results of PCB and pesticide residues in foods", Annu. Rep. Osaka Pref. Inst. Publ. Health (fiscal year 1992), 1993, p. 86-87.

9) Kuwabara, K., Matsumoto, H., Murakami, Y., Hori, S., Daily dietary intakes of PCBs and organochlorine pesticides during 19 years from 1977 to 1995 by adults in Osaka evaluated by the total diet study method. Shokuhin Eiseigaku Zasshi (J. Food Hyg. Soc. Japan), 38, 286-295 (1997).

10）東京都立衛生研究所毒性部病理研究科, 内分泌汃乱作用 が疑われる化学物質の生体影響デー夕集，1999, p. 13-22, p. 67-132.

11) vom Saal, F. S., Timms, B. G., Montano, M. M., Palanza, P., Thayer, K. A., Nagel, S. C., Dhar, M. D., Ganjam, V. K., Parmigiani, S., Welshons, W. V., Prostate enlargement in mice due to fetal exposure to low doses of estradiol or diethylstilbestrol and opposite effects at high doses. Proc. Natl. Acad. Sci. USA. 94, 2,056-2,061 (1997).

12）日本薬学会編，“衛生試験法・注解 1990”, 東京, 金原出 
版(株), 1990, p. 618-626.

13) Ugawa, M., Nakamura, A., Kashimoto, T., Studies on a calculation method for polychlorinated biphenyl (PCB) isomers. Shokuhin Eiseigaku Zasshi (J. Food Hyg. Soc. Japan), 14, 415-424 (1973).

14) Araki, S. et al. eds., "Kankyo Kagaku Jiten", Tokyo, Tokyo Kagaku Doujin Co., 1985, p. 656.

15) Muto, S., "Noyaku Gaisetsu (Outline of Pesticides)", Tokyo, Gihodo Shuppan Co., 1970, p. 805.

16) Muto, S., "Noyaku Gaisetsu (Outline of Pesticides)", Tokyo, Gihodo Shuppan Co., 1970, p. 724.

17) Araki, S. et al. eds., "Kankyo Kagaku Jiten”, Tokyo,
Tokyo Kagaku Doujin Co., 1985, p. 535.

18) Stellman, S. D., Djordjevic, M. V., Britton, J. A., Muscat, J. E., Citron., M. L., Kemeny, M., Busch, E., Gong., L., Breast cancer risk in relation to adipose concentrations of organochlorine pesticides and polychlorinated biphenyls in Long Island, New York. Cancer Epidemiol. Biomarkers Prev., 9, 1,241-1,249 (2000).

19) Pavuk, M., Cerhan, J. R., Lynch, C. F., Kocan., A., Petrik, J., Chovancova., J., Case-control study of PCBs, other organochlorines and breast cancer in Eastern Slovakia. J. Expo. Anal. Environ. Epidemiol., 13, 267-275 (2003). 